\title{
Breakage of Automotive Spring Washer during Twist Test: A Metallurgical Analysis
}

Das $\mathbf{S}^{1 *}$, Talukder $\mathbf{S}^{2}$, Solanki $\mathbf{V}^{1}$, Adhikary $\mathbf{M}^{1}$ and Bhattacharya $\mathbf{S}^{1}$

${ }^{1} R \& D$ and Scientific Services, Tata Steel Limited, Jamshedpur 831 001, Jharkhand, India

${ }^{2}$ Technology Group (Long Products \& Global Wires), Tata Steel Limited, Jamshedpur, Jharkhand, India

\begin{abstract}
A washer is a thin plate with a hole that is normally used to distribute the load of a threaded fastener, such as a screw or nut. The washer used in automotive engine component is of split or spring lock washer. A ring split at one point and bent into helical shape. The benefit of spring lock washers lies in the trapezoidal shape of the washer. When compressed to loads near proof strength of the bolt, it will twist and flattern. This reduces the spring rate of the bolted joint which allows it to maintain more force under the same vibration levels. This prevents from loosening. The steel washer is one of the critical safety component for automobile engine and its failure may cause severe safety issue. Before subjecting to manufacturing the washer undergoes different process lines (Wire Rod $\rightarrow$ Drawing $\rightarrow$ Annealing $\rightarrow$ Pickling $\rightarrow$ Flattening $\rightarrow$ Spring Washer $\rightarrow$ Single Spring Washer). After manufacturing of washer toughness test is done to find out the metallurgical soundness and surface quality of the washer. In this toughness test the washer in a vice with the split ends free and straight above the vice jaws, a $90^{\circ}$ segment of the free end is gripped with a wrench and bent. Washers should withstand twist test through a $90^{\circ}$ angle without signs of fracture. The present paper highlights premature failure of automotive washer which failed during twist test. From the analysis, it has been observed that the distribution of spheroidized carbide is more uniform in good samples than that of rejected samples. Hardness profile variation was observed between good and bad spring samples. Overall investigation does not indicate any problem related to supplied wire rod material quality as no wire breakage problem observed Inhomogeneous annealed structure in flat wire seems to result into variation in washer performance during twist test.
\end{abstract}

Keywords: Spring lock washers; Automobile; Twisting; Spheroidized carbide; Annealing

\section{Introduction}

A washer is a thin plate (typically disk-shaped) with a hole (typically in the middle) that is normally used to distribute the load of a threaded fastener, such as a screw or nut. Other uses of washer could be a spacer, spring (belleville washer, wave washer), wear pad, preload indicating device, locking device, and vibration reducing element (rubber washer). Washers usually have an outer diameter (OD) about twice larger than their inner diameter (ID). The washer used in automotive engine component is of split or spring lock washer. A ring split at one point and bent into helical shape. The benefit of spring lock washers lies in the trapezoidal shape of the washer. When compressed to loads near proof strength of the bolt, it will twist and flattern. This reduces the spring rate of the bolted joint which allows it to maintain more force under the same vibration levels. This prevents from loosening $[1,2]$. The steel washer is one of the critical safety components for automobile engines and its failure may lead to severe safety issue. Before subjecting to manufacturing, the washer undergoes processing steps from rolling to flattening operations (Figure 1). During processing, breakages can occur, causing operational interruptions and decrease in productivity. The types of breakages that occur during washer making may be attributed to improper metallurgical quality of the input wire rod/ wire, inadequate lubrication, improper die profile, excessive reduction in cold drawing, and other material/operational factors. The pipe, porosity, entrapment, and segregation in the input wire rods must also be within tolerable limits for suitable washer making. Additionally, wire rods that are heat treated for subsequent drawing must be of suitable microstructure, dimensionally appropriate, and free from surface defects such as laps, seams, and fins and micro flaws. Ideally, a fine pearlitic microstructure is desirable in wire rods/wires subjected to patenting heat treatment, because this microstructure is most amenable for sequential reductions in cold drawing. In a modern rolling mill a fine pearlitic microstructure is achieved by laying the rings of wire rod thinly on a conveyor and subjecting to forced cooling of the wire rods by blowing strong blast of air through these rings. In actual industrial practice, however, undesirable microstructures such as coarse pearlite, bainite, martensite may develop as a result of improper heat treatment during rolling or during post operation like patenting (e.g., inadequate/excessive soaking during austenitization, high/low lead bath temperature, etc.) [3]. Sometimes foreign materials
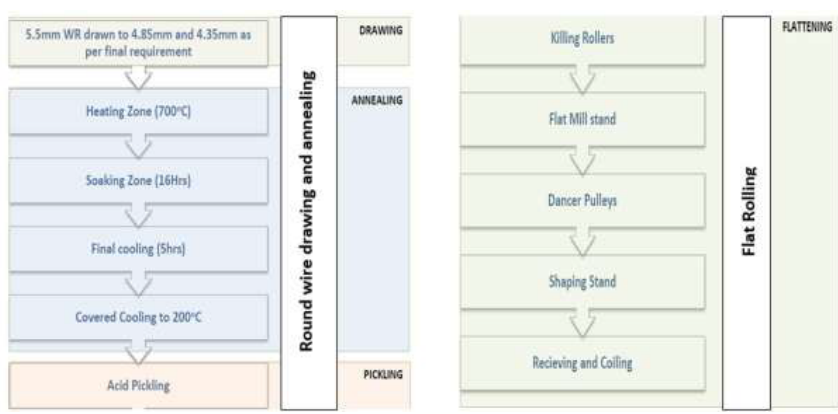

Figure 1: Processing steps from rolling to flattening operations of washer

*Corresponding author: Souvik Das, R\&D and Scientific Services, Tata Stee Limited, Jamshedpur 831 001, Jharkhand, India, Tel: +91-8092084714; E-mail souvik.das@tatasteel.com

Received December 08, 2017; Accepted December 12, 2017; Published December 13, 2017

Citation: Das S, Talukder S, Solanki V, Adhikary M, Bhattacharya S (2017) Breakage of Automotive Spring Washer during Twist Test: A Metallurgical Analysis. J Steel Struct Constr 3: 136. doi: 10.4172/2472-0437.1000136

Copyright: (c) 2017 Das S, et al. This is an open-access article distributed under the terms of the Creative Commons Attribution License, which permits unrestricted use, distribution, and reproduction in any medium, provided the original author and source are credited. 
can get entrapped in the wire rod during rolling operation which may lead to improper structure. Thus undesirable structures certainly impair draw ability and may lead to wire breakages during subsequent cold drawing operations. Die lubrication is also an important factor in cold drawing because wire breakages are often caused by lubrication breakdown on the wire surface. Lubrication breakdown may result in localized generation of heat due to direct wire-to-die contact [2]. In this present work, a microstructural study of broken washer samples was conducted to determine the genesis of failure.

\section{Experimental Details}

A $1.5 \mathrm{~mm}$ diameter flat drawn washer samples was taken for the study. This found broken during twist test. Material was basically from high carbon wire rod grade. Broken wire samples that underwent breakage at the time of testing had been processed from high carbon ( 0.70 to 0.85 wt. $\%$ C), $130 \times 130 \mathrm{~mm}$ square billets. Details of the broken washer samples are provided in Table 1.

\section{Sample preparation}

Two pieces of washer that had broken during drawing and two flat samples were collected. The samples were cleaned with acetone to remove dirt and other adherent debris for visual examination prior to fractography and metallographic sample preparation. Transverse and longitudinal specimens were prepared from the fractured end of each failed washer samples for conducting light microscopic examination. These samples were individually mounted in conductive copper-containing resin and polished by conventional metallographic techniques. Failed surface was examined under upright metallurgical microscope under un-etched and etched condition. Etching was done by $3 \%$ of Nital.

\section{Visual observation}

Failed samples were visually observed under macro dual zoom microscope Figure 2a. The nature of broken end indicated that the failure took place during twisting. The fracture surface of the broken spring washer revealed shiny granular appearance with chevron marks indicating brittle fracture (Figures $2 \mathrm{~b}$ and $2 \mathrm{c}$ ).

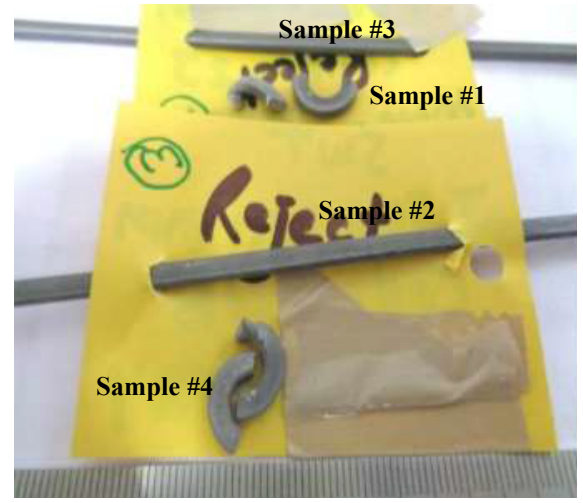

Figure 2a: Overall view of samples referred for analysis.

\section{Fractography}

The fracture surface of the failed sample \#1 was observed under Field Emission Gun Scanning Electron Microscopy (FEG-SEM) (Figure 3). Fractography of the facture surface (sample \#1) revealed cleavage nature of the fracture surface indicating brittle fracture.

\section{Microstructural analysis}

The failed sample was etched with 3\% Nital solution. Etched microstructure revealed presence of spheroidized carbide in ferrite matrix. In both the failed spring washers, non/uniform spherodisation was observed in Figures $4 \mathrm{a}$ and $4 \mathrm{~b}$ without any carbide network.

\section{Heat treatment (HT)}

The flat wire samples ( 3 and 4 ) of the failed lots were subjected to heat treatment to find out the effect of the heat treatment cycle. First the component was heated above austenizing temperature at around $850^{\circ} \mathrm{C}$, followed by cooling at a temperature below the eutectoid temperature $700-710^{\circ} \mathrm{C}$. It was then hold for a prolonged period (22-25 Hours) and furnace cooling was done at a very slow rate with appropriate soaking time (Figure 5a). After heat treatment distribution of spheroidized carbide was found more uniform in spring washer samples after heat treatment than that of rejected samples (Figures $5 \mathrm{~b}$ and $5 \mathrm{c}$ ).

\section{Hardness measurement}

The micro hardness at different locations of the samples was measured (before and after heat treatment) in a pneumatically controlled automatic micro hardness tester (Leco-LM247AT). An applied load of $5 \mathrm{~kg}$ was used during testing, and several indentations

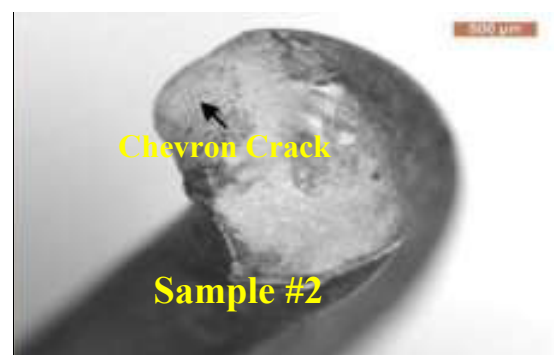

Figure 2b: Fracture surface of the twisted sample.

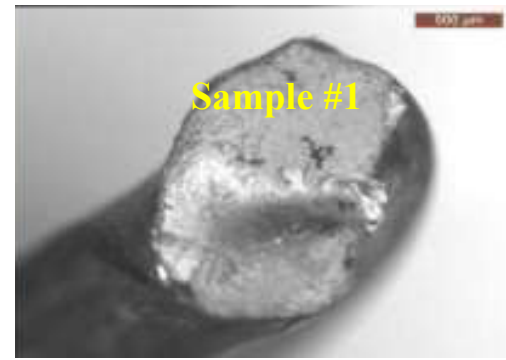

Figure 2c: Fracture surface of the twisted sample.

\begin{tabular}{|c|c|c|c|c|c|c|c|c|c|}
\hline \multirow[t]{2}{*}{ SI. No } & Sample Type & \multicolumn{7}{|c|}{ Chemical Composition (wt \%.) } & \multirow[t]{2}{*}{ Section $(\mathrm{mm})$} \\
\hline & \multirow{3}{*}{ Washer } & C & Mn & $\mathbf{S}$ & $\mathbf{P}$ & $\mathbf{S i}$ & $\mathrm{Cr}$ & N2 ppm & \\
\hline 1 & & 0.738 & 0.7 & 0.018 & 0.026 & 0.19 & 0.015 & 40 & $1.59 / 1.60$ \\
\hline 2 & & 0.74 & 0.7 & 0.017 & 0.023 & 0.18 & 0.014 & 35 & $1.58 / 1.61$ \\
\hline 3 & \multirow[t]{2}{*}{ Flat } & 0.739 & 0.72 & 0.017 & 0.022 & 0.18 & 0.014 & 37 & $1.59 / 1.62$ \\
\hline 4 & & 0.74 & 0.71 & 0.017 & 0.024 & 0.18 & 0.014 & 36 & $1.59 / 1.63$ \\
\hline
\end{tabular}

Table 1: Details of failed washer samples along with flat samples. 
Citation: Das S, Talukder S, Solanki V, Adhikary M, Bhattacharya S (2017) Breakage of Automotive Spring Washer during Twist Test: A Metallurgical Analysis. J Steel Struct Constr 3: 136. doi: 10.4172/2472-0437.1000136

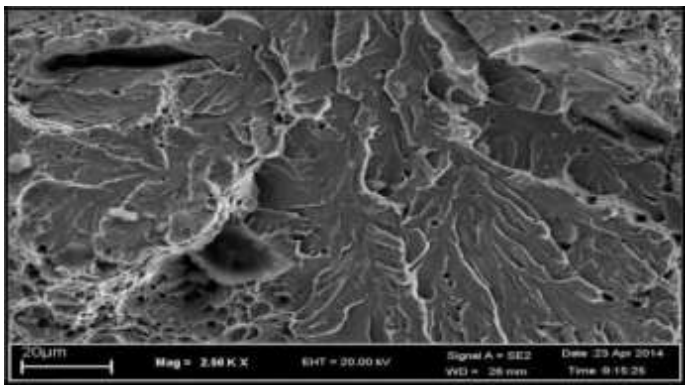

Figure 3: Fractography analysis of sample \#1 of the failed component.

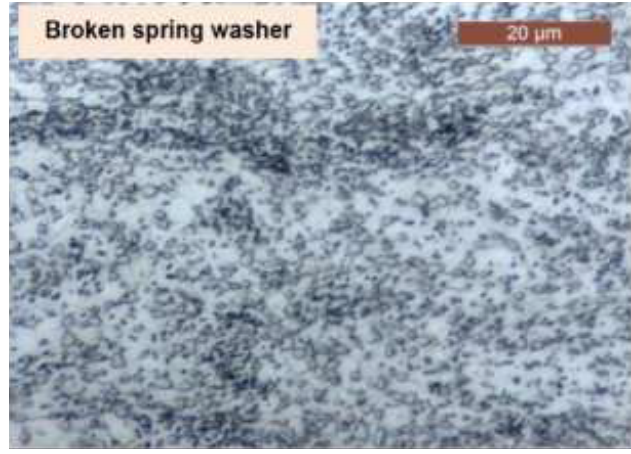

Figure 4a: 3\% Nital Etched microstructure of failed twisted Sample 1.

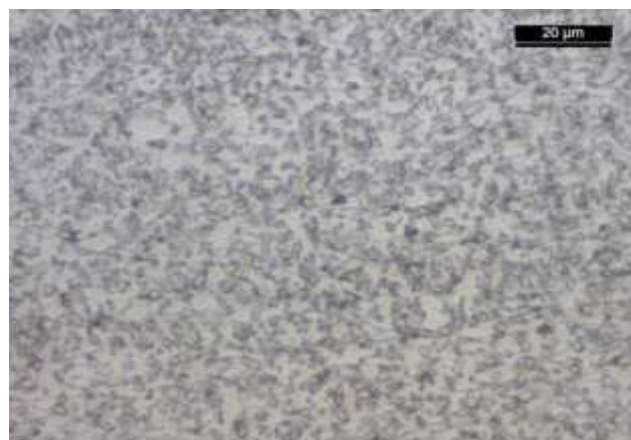

Figure 4b: 3\% Nital Etched microstructure of failed twisted Sample 2

were made to determine the hardness of the flat samples (before and after heat treatment).The hardness of the samples before heat treatment was found higher with respect to after heat treatment (Table 2).

\section{Twist Test}

Twisting operation is done as per IS 6755 specifications [4]. In case of twist test a portion of the spring washer is gripped in vice jaws and then equal portion is gripped in wrench jaws as shown in Figure 6a. The wrench is then rotated in a direction that increases the free height of the spring washer till the washer is twisted through an angle of $90^{\circ}$. It is slowly and evenly bent (not jerkily) taking precaution to prevent injuries through fly splitters Figures $6 \mathrm{~b}$ and $6 \mathrm{c}$. In case of "after heat treatment" sample no fracture was observed during the twist test (Table 3).

\section{Discussion}

1. The nature of breakage indicated that the failure took place during twisting.

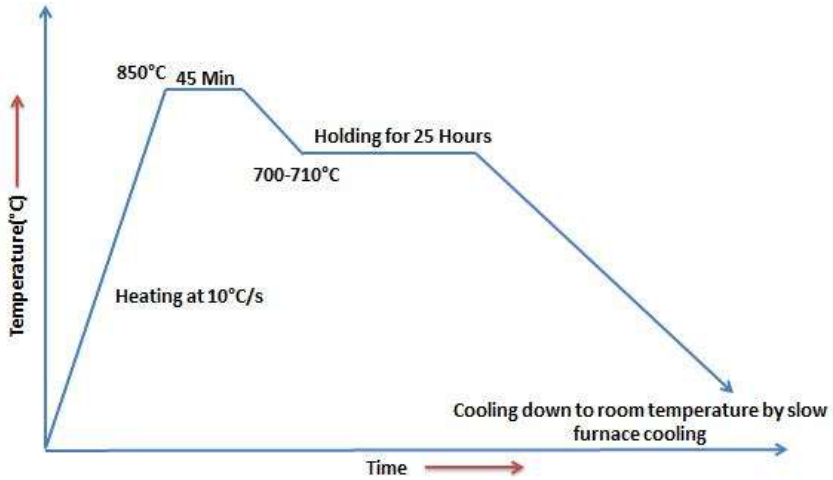

Figure 5a: Heat Treatment cycle.

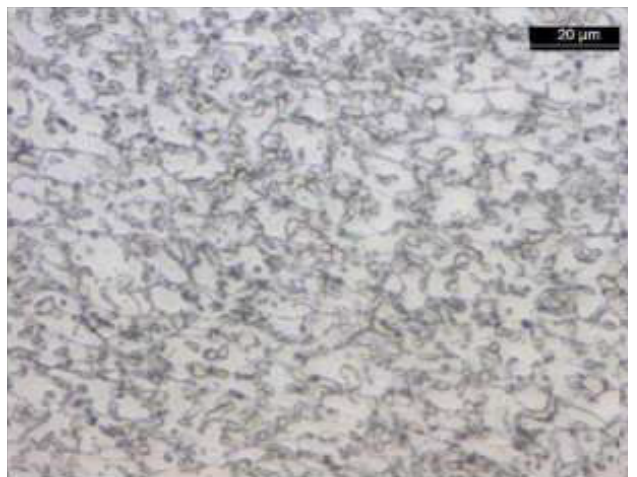

Figure 5b: $3 \%$ Nital Etched microstructure of flat failed samples before hea treatment.

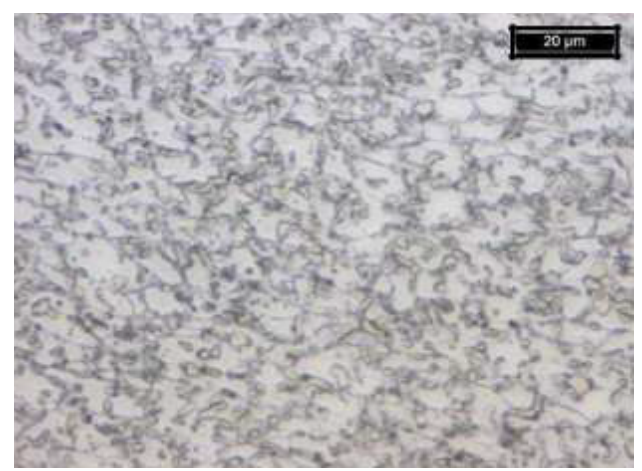

Figure 5c: $3 \%$ Nital Etched microstructure of flat failed samples after heat treatment.

\begin{tabular}{|c|c|c|}
\hline Spec & Sample type & Hardness (HV-5 kg) \\
\hline $\mathbf{1}$ & Flat Sample \#3 (Before HT) & $27,12,64,250$ \\
\hline $\mathbf{2}$ & Flat Sample \#3 (After HT) & $22,32,24,227$ \\
\hline
\end{tabular}

Table 2: Details of hardness of the flat samples (before and after heat treatment).

\begin{tabular}{|c|c|c|}
\hline Spec & Sample type & Twisting test \\
\hline $\mathbf{1}$ & Washer Sample \#3 (Before HT) & Fracture \\
\hline $\mathbf{2}$ & Washer Sample \#3 (After HT) & No Fracture \\
\hline
\end{tabular}

Table 3: Details of twist test.

2. The fracture surface of the broken spring washer revealed shiny granular appearance with chevron marks indicating brittle fracture. 


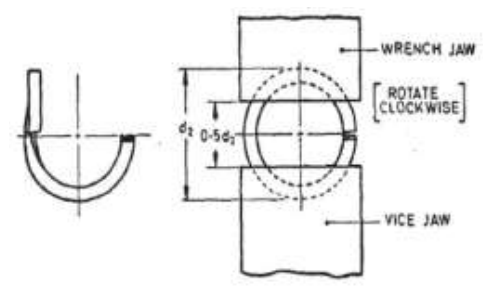

Figure 6a: Twist Test.

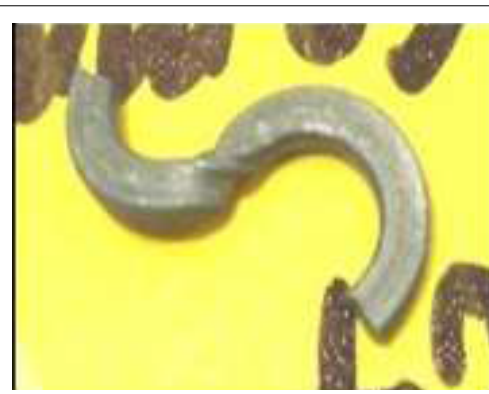

Figure 6b: Overall view of sample before heat treatment

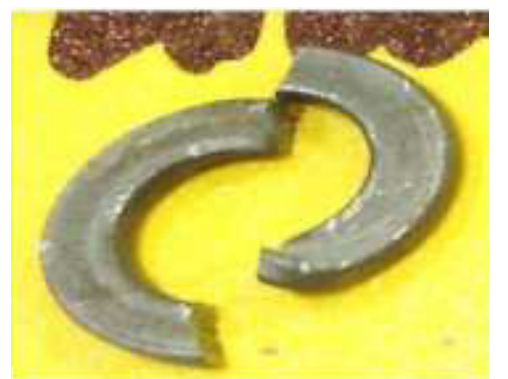

Figure 6c: Overall view of sample after heat treatment.
3. Microstructural analysis of both the failed spring washer sample shows non-uniform spherodisation structure. This indicates improper spherodise annealing cycle was followed.

4. Spheroidizing of high carbon steel is a method of prolonged heating at a temperature below the eutectoid temperature. By heating at this temperature pearlite, which is the lowest energy arrangement of steel, gets converted to ferrite and cementite. The graphite content of steel assumes a spheroidal shape after spheroidizing and after prolonged heating the pearlite layers are broken down and spherical lumps of cementite, or spheroidite, are formed [5-7].

5. Due to the abnormality in the heat treatment process of the flat wire samples non-uniform spherodisation structure was formed which resulted in high hardness of the sample. Due to the high hardness, the washer samples failed during twist test.

\section{Conclusion}

Inhomogeneous spherodised annealed structure in flat wire resulted into variation of spring washer performance during twist test.

\section{References}

1. Das S, Koli P, Mathur J, Dey A, Bhattacharyya T, et al. (2013) Failure analysis of Motor Tyre Bead Wires during Torsion Test. Engineering failure Analysis and Prevention, pp: 684-688.

2. ZHANG YF, CHEN L, LIU YW (2012) Failure Analysis of 65Mn Spring Washer Fracture [J]. Failure Analysis and Prevention 7: 43-45.

3. Das S, Mathur J, Bhattacharyya T, Bhattacharyya S (2013) Metallurgica investigation of different causes of centre bursting led to wire breakage during production. Case Study of Engineering Failure Analysis 1: 32-36.

4. IS: 6755-1980: Specification of double coil helical spring washers

5. Krauss G (2015) Steels: processing, structure, and performance. Asm International, pp: 256-259.

6. Totten GE, Howes MAH (1997) Steel Heat Treatment Handbook. New York, NY: Marcel Dekker, p: 540.

7. O'Brien JM, Hosford WF (1997) Spheroidization of medium-carbon steels. Journal of Materials Engineering and Performance 6: 69-72. 